Article

\title{
Narratives of Psychosocial Response to Microtrauma Injury among Long-Distance Runners
}

\section{Hayley C. Russell ${ }^{1, *}$ and Diane M. Wiese-Bjornstal ${ }^{2}$}

1 Department of Kinesiology, The Pennsylvania State University, Altoona Campus, Altoona, PA 16601, USA

2 School of Kinesiology, University of Minnesota, Twin Cities, Minneapolis, MN 55455, USA; E-Mail: dwiese@umn.edu

* Author to whom correspondence should be addressed; E-Mail: hcr3@psu.edu; Tel.: +1-814-949-5639.

Academic Editor: Arno Schmidt-Trucksäss

Received: 2 June 2015 / Accepted: 24 July 2015 / Published: 30 July 2015

\begin{abstract}
Athletes with microtrauma or overuse injuries resulting from an accumulation of repeated small forces may differ from athletes with macrotrauma or acute injuries in their psychosocial responses because of the unique challenges presented by these insidious-onset and often chronic injuries. Our purpose was to use narrative inquiry to examine the psychosocial experiences and responses of 10 long-distance runners who had experienced microtrauma injuries. Qualitative data analysis of interview data led to a chronological timeline of the injury experience and an assessment of the meaning attributed to these injury experiences using a variation of Mishler's core-narrative approach. Participants reported distinct thoughts, feelings, and behaviors during each phase of the injury-pre-injury, injury onset, and outcome. In the pre-injury period, participants indicated specific running-related goals and attributed their injuries to overtraining or a change in training. During the injury onset phase, participants consistently indicated two themes: self-diagnosis and treatment, and not taking time off. Within the outcome phase of injury, participants acknowledged changed training because of the injury, and lessons learned from their injury experiences. The narratives of microtrauma-injured runners revealed psychosocial distress and behavioral tendencies post-injury that have important implications for runners, coaches, and healthcare professionals.
\end{abstract}


Keywords: injury; running; chronic injury; sport psychology

\section{Introduction}

Injuries are relatively common consequence of long-distance running. Researchers have found that 79\% of long-distance runners will experience an injury during one year of training [1]. Long-distance runners who experienced a disruption in training due to a sport injury reported a variety of psychosocial symptoms such as depression and anxiety as well as endorsed low self-esteem and feelings of confusion and frustration [2]. Psychosocial refers to thoughts, feelings, and behaviors experienced by injured runners, as well as the social context in which these responses occur. In line with predictions of the integrated model of psychological response to sport injury [3], these findings provide evidence that long-distance runners experience potentially negative and distressing psychosocial responses to the sport injury process. Given the increased interest in long-distance running in the United States and the associated risk for injury, long-distance runners are an important subset of athletes to study to better understand the chronology of events as well as the psychosocial responses to injuries.

Individuals' responses to sport injuries can vary and, according to Wiese-Bjornstal [4], both personal and situational factors affect athletes' cognitive, emotional, and behavioral responses to sport injuries. Injury type is one of the personal factors suggested to influence an individual's responses to sport injury [3]. Injuries are typically categorized as one of two types-macrotrauma or microtrauma. Macrotrauma injuries result from an isolated, sudden impact or force (e.g., strains, sprains, dislocations, and fractures), whereas microtrauma injuries occur as a result of the accrual of repeated small forces over a period of time (e.g., stress fractures, tendinitis) [5]. The majority of evidence suggests that longdistance runners are more prone to microtrauma injuries than macrotrauma injuries. In a systematic review, van Gent and colleagues [6] found that the incidence of lower extremity running injuries was as high as 79.3\% during the reporting period for studies reviewed (ranging from one day to 18 months); most of which were microtrauma in nature. Consistent with the integrated model of psychological response to sport injury [3], Flint [5] suggested that due to the differences in the onset of injury, psychosocial responses of individuals incurring microtrauma injuries might differ from those of individuals who experience macrotrauma injuries. For instance, individuals dealing with microtrauma injuries report a period of gradually increasing pain accompanied by decreased athletic performance during onset [5]. Due to the insidious onset and longevity of microtrauma injuries, athletes may experience significant and prolonged emotional distress (e.g., frustration, sadness), whereas the sudden onset of macrotrauma injuries allows athletes to immediately deal with the injury and rehabilitation process [5].

\section{Context}

Previous research has supported the suggestion by Flint [5] that differences exist in the psychosocial responses to microtrauma injuries and macrotrauma injuries. In particular, Wasley and Lox [7] found that athletes who experienced a microtrauma injury scored more negatively on measures of self-esteem, sought social support less, and reported more avoidance or escape behaviors than athletes who incurred 
a macrotrauma injury. Although limited by a small sample size and only presenting preliminary results, the findings suggest that the type of injury an athlete experiences may influence the nature of psychosocial response to a sport injury. Concerning social support, Henert [8] found that individuals with microtrauma injuries rated most types of social support as being more important to their overall well-being than individuals with macrotrauma injuries. These studies highlight two key points. First, psychosocial responses to injury differ based on the type of sport injury an individual experiences. Second, athletes report a discrepancy in the value of social support such that individuals with microtrauma injuries identify social support as being more important to overall well-being [8]; however, these individuals tend to seek social support to a lesser degree than individuals who experience a macrotrauma injury [7]. Because of the limited studies examining microtrauma injuries as well as the discrepancy in findings regarding psychosocial responses based on injury type, the current study was exploratory in nature, to better understand the process of psychosocial responses to microtrauma sport injuries.

Due to the complex and multi-dimensional nature of psychosocial responses to sport injuries [3], it is important to understand the full experiences of injured athletes. To date, researchers have primarily examined the psychosocial responses of athletes to sport injuries using quantitative methods or semi-structured interviews [9-12]. Another type of qualitative research that can be used to better understand lived experiences of athletes recovering from a sport injury involves narrative research methods; however, these methods have rarely been used in examining the experience of injured athletes. Narrative research methods are effective in studying an individual's experiences over time [13] and provide “coherence and continuity to one's experience” [14]. Thus, narrative research methods may provide a full picture of the injury and recovery process over time. By using this research method with long-distance runners incurring microtrauma injuries, we gain a fuller understanding of the unique aspects of these types of injuries that have been underrepresented in the psychology of sport injury literature to date.

Narrative research methods have been used to better understand the psychosocial responses of individuals with health-related issues [15] and with individuals recovering from non-sport related injuries [16]. In general, narrative research methods allow individuals to provide more thorough, less researcher-directed discussions of psychosocial responses to health issues or injuries as compared to quantitative research methods using self-report questionnaires or even semi-structured interviews. Thus, narrative research methods may help researchers identify responses that have been overlooked by other methods. With respect to sport injuries, Brock and Kleiber [10] used narrative research methods to examine athletes who suffered career-ending injuries 20 or more years prior to the investigation. The authors found that sport injuries inevitably led to a mild-to-severe change in both identity and self-esteem. Brock and Kleiber found a regular pattern within the stories that the injured athletes shared. Specifically, each athlete's story always included a section on the athlete's emotional response (e.g., feelings of confusion, isolation, anger) that resulted from experiencing a loss associated with the injury. Based on information derived from narrative research methodology in health-related research and sport injury research, we believe that the use of narrative research methodology is a valuable way to gain knowledge of the full experience of athletes who have experienced a microtrauma sport injury.

The use of narrative research methods in the present study fills a gap in research by providing a better understanding of the psychosocial responses (defined as thoughts, feelings, and behaviors) that 
individuals experience during microtrauma injuries. Thus, the purpose of the current study was to use narrative inquiry and analysis to examine the distinct experiences and responses of long-distance runners who had recently experienced microtrauma injuries. Results are reported for two main research questions:

1. What psychosocial responses do long-distance runners experience surrounding a microtrauma injury?

2. What is the chronology of psychosocial experiences associated with microtrauma injuries in long-distance runners?

\section{Method}

\subsection{Participants}

A purposeful sample of runners was chosen. This sample was selected because they were able to provide useful information about the phenomenon of study, specifically, microtrauma running injuries [17]. Participant recruitment continued until data saturation was reached. Ultimately, 10 participants (eight females, two males) between the ages of 19 and 37 years of age $\left(M_{\text {age }}=25.1\right)$ were recruited from a local running store and a long-distance running group at a large Midwestern university to participate in this study (see Table 1 for demographic and participant injury information). Participants were recruited to participate in this study if they met the following inclusion criteria. Participants must have suffered a microtrauma (defined, for the purposes of the current study, as an injury occurring due to the accrual of repeated small forces over a period of time as a result of long-distance running training) [5] within the 18 months prior to recruitment. Participants' injuries were self-reported. Participants also had to be competitive runners, defined for the purpose of this study as having trained for and were planning to, or had run, in at least one five-kilometer race in the preceding 12 months. Participants varied from novice to experienced runners. Additionally, participants had to be between the ages of 18 and 64 at the time of the interviews. Pseudonyms were used for all participants in order to maintain anonymity and confidentiality.

Table 1. Participant characteristics.

\begin{tabular}{cccc}
\hline Pseudonym & Gender & Age & Self-Reported Injury \\
\hline Logan & Male & 22 & Sciatic nerve; shin splints \\
Anne & Female & 37 & Pulled hamstring/illiotibial band; skin pain \\
Grace & Female & 23 & Achilles tendonitis; stress fracture \\
Caroline $*$ & Female & 22 & Achilles tendonitis; knee patella femoral \\
Jordan & Female & 22 & Pain on inner right foot; illiotibial band \\
Laura & Female & 21 & Patella subluxation (knee) \\
Jane & Female & 19 & Knee pain, Sacroiliac pain \\
Brad & Male & 21 & Separated shoulder; tibia stress fracture \\
Maryn & Female & 25 & Quadriceps strain; pulled gluteus medius \\
Kathryn & Female & 19 & Illiotibial band; blisters \\
Cynthia & Female & 20 & Runner's knee (right and left); baby fracture \\
\hline
\end{tabular}

Note: *No audio or video data. Participant's data not include in data analysis. 


\subsection{Procedure}

Institutional review board (IRB) approval was obtained prior to participant recruitment. In order to recruit individuals to participate in this narrative study, advertisements were posted at a local running store and an advertisement was made to a running group at a local university. Advertisements directed participants to contact the principal investigator (PI), by either phone or email, if s/he was interested in participating in the study. Additionally, the advertisements indicated that participants would receive a gift card for a local running store for participating in the study. Upon initial contact with the PI potential participants were asked questions related to the inclusion criteria for the study to determine if they qualified to participate in the study. If they met the inclusion criteria for the study, participants met with one of the researchers to complete the interview. Once the informed consent had been completed, the participants began the interview. Face-to-face interviews were conducted with all participants. Interviews ranged in time from 11 to $33 \mathrm{~min}$. Participants were asked to tell the story of their injury, including events that took place immediately prior to the injury (henceforth referred to as pre-injury factors), the injury itself, and what happened after the injury. Participants were given this interview prompt verbally and in written form to refer to as they told their story. The only specific questions asked by the interviewers were probes to provide further clarification or elaboration on information provided by the participants. All interviews were video- and audio-recorded. Video recording allows the researchers to observe participant's body language, facial expressions, and other idiosyncrasies associated with the communication of the narrative. In addition, this technique aids the researchers in providing consistency during data analysis and coding, as well as the ability to contribute relevant observational comments, which are critical supporting evidence for the research [17].

\subsection{Data Analysis}

The interviewers transcribed each interview verbatim. The researchers then examined video transcripts of the interviews to add additional detail to the transcripts (e.g., crying). As per the example provided by Brock and Kleiber [11] in their modified narrative analysis technique, transcripts were prepared by numbering each new topic as the conversation progressed, for example:

075: P: Um, I’ve talked to my roommate a lot. She’s one of my best friends and I, you know, through talking to her like, I don't want to, like have a pity party about it.

076: so I’ve just kind of, um, come to the realization that, like, I will finish. Like I will probably have to walk some, like I will...it will tack another hour.

Additionally, two different types of statements from the transcript were transferred to two new transcripts: the core narrative and evaluative commentary. The first transcript, labeled core narrative included any statement that contributed to the understanding of the sequence of events relating to the injury. For example statements such as “026: P: Umm I was doing a 5k and it, my leg just all of a sudden started, well, my left butt cheek started hurting it was a throbbing pain, I could literally not run, I would have to walk" were located in the core narrative transcript. The second type of transferred statement was any statement that contributes to the understanding of the sequence of events relating to the injury. This 
transcript was called the evaluative commentary. Statements such as "052: but it lingered on for so long, then it was kind of feelings of what is this and is it ever going to go away" and "053: at times it was feelings of, umm, it was frustration” were located in the evaluative commentary transcript.

Both the core narrative and evaluative commentaries were analyzed for common themes relating to the two primary research questions for this study. Specifically, the core narrative transcripts were analyzed to examine consistency and divergence in the chronology of the experience of athletes with microtrauma injuries. The evaluative commentary and core narrative transcripts were analyzed for common themes relating to the psychosocial response to the injuries as well as the meaning attributed to the injuries. Data analyses were similar to interpretative phenomenological analysis wherein the lived experiences of participants and the meaning attributed to the experiences are examined [18] and following the example provided by Brock and Kleiber [10]. Analyses consisted of the following steps. First, two researchers prepared the core narrative and evaluative commentary transcripts [10] and then reviewed the core narrative transcripts independently. This step involved several thorough readings of the transcripts identifying points of interest [18]. Second, researchers independently identified common statements between participants relating to the psychosocial responses to injury and the chronology of events associated with the injury. Third, researchers clustered common statements into overarching themes. Fourth, researchers compared the independently identified themes for consistency and divergence between researchers. Fifth, transcripts were then re-examined by the researchers to settle any disagreements in emergent themes leading to the final themes relating to the psychosocial responses to injury and the chronology of events. Another researcher then reviewed final themes after reading and re-reading the transcripts and was in agreement with the emergent themes [19].

\section{Results}

Participants' stories revealed that their experiences with running injuries had a meaningful impact on their lives. Analysis of the narrative data identified psychosocial responses to injury within the participants' stories and a consistent chronology of events and responses associated with injuries. In telling their stories, participants identified common experiences and responses during the pre-injury, injury, and post-injury phases of their injury process

\subsection{Research Question 1: Psychosocial Responses}

In discussing their injuries, participants revealed consistent psychosocial responses to injury. These themes were consistent cross the injury and recovery process. Overwhelmingly, the participants' responses to their injuries were negative throughout the injury process. Participants noted responses such as being "devastated", "bummed out”, and "panicked” by the injury. The psychosocial distress experienced by the participants divided into four overarching sub-themes-frustration, fear, general emotional distress, and social influences.

Frustration. Microtrauma injuries for runners were described as frustrating. This frustration seemed to be due to the uncertainty or ambiguity associated with microtrauma injury. As previously discussed, microtrauma injuries have an insidious onset and can persist for a long period of time, yet may not be perceived as serious as macrotrauma injuries [5]. These characteristics that distinguish microtrauma injuries from macrotrauma injuries seemed to contribute to the frustration experienced by participants. 
For example, Anne described the unpredictable nature of microtrauma injuries and perceptions of injury severity as contributing to her frustration.

"I think a little bit of frustration", she said in describing her feelings associated with the injury,

“because every time I would go out running it would hurt and I didn’t want it to hurt but it didn’t hurt enough to stop necessarily.”

Similarly, Grace noted that the unpredictable nature of microtrauma injuries contributed to her frustration, and she said,

"It like kind of feels like a rubber band, you know. ...it reappears just randomly and there wasn't much of a pattern so it would just, it would just get really frustrating.”

Even more specific to the nature of microtrauma injuries, Maryn specifically compared the experience with her microtrauma injury to that of a macrotrauma injury in terms of the frustration she experienced. She said,

“Even though they're [running injuries] not as extreme as tearing your ACL, they're nagging injuries. And they're kind of... sometimes more irritating because they're not concrete. You can't go in and say ‘yea, you have an ACL tear, you need to do this, this, and this.' You've got a muscle strain ... you need to just rest”.

Generally, the frustration experienced by participants was closely related to the insidious onset, persistent symptoms, and unpredictable nature of microtrauma injuries.

Fear. In addition to frustration, participants also indicated they experienced a great deal of fear associated with their injuries. The theme of fear subdivided into four distinct lower-order themes-fear of not recovering, fear of re-injury, fear of diminished performance, and fear of appearing weak.

Fear of not recovering. In addition to the frustration participants were experiencing in response to the ambiguity in their injury, participants also indicated they feared the uncertainty of the diagnosis and the unpredictability of the recovery, again characteristics that differentiate microtrauma injuries from macrotrauma injuries. Jordan noted experiencing fear about a number of different factors associated with her injury and further demonstrated her fear by identifying some injury-related catastrophic thinking:

"I really was freaked out about like is this a major foot injury? Am I going to get a stress fracture or something? Like, is this going to prevent me from running the marathon like 5 months later?”

Anne echoed the sentiment of fear of the injury not going away. She said,

"But it was also like a little bit scared, like what is going on and why is this lingering on for so long and why is my foot still swollen and it was just weird. So, 'cause it usually, nothing's ever lasted that long for me unless it was broken.”

Kathryn indicated that the most common emotion she felt during her injury was fear or nervousness as she described it: 
"I think the biggest thing I felt when I had it [the illiotibial (IT) band injury] was nervousness, because I was really scared.”

She also indicated that part of her fear was associated with the uncertainty of her injury saying that,

"I just didn’t really know what was happening."

Fear of diminished performance. Most participants in this study were training for a particular race or event when they became injured and feared being unable to meet their goals related to the event, whether they were a specific performance standard or completing the event. For example, Laura was scared of not being able to complete her upcoming marathon:

"I was like kind of freaking out because I was like, I'm not going to be running so my training, I'm gonna get behind and then I'm not going to be able to run the marathon.”

Similarly, Jordan indicated fear in getting behind in her training, stating:

"I just kind of panicked because, you know, we're over half way done training, the marathon's like a month and a half away.”

Through tears, recalling her injury, Jane said,

“But, it's like I've trained healthy and now, like, the most I've ever run on it is two hours and that was absolutely brutal. And, it's just...I'm just worried that I'm not like physically not going to be able to finish and, that's just absolutely devastating to me right now."

Fear of re-injury. Fear continued to be a prominent theme even after the runners thought they had recovered from their injuries. Overwhelmingly there was a sense of relief among participants once they felt they had recovered from the injury. However, entwined with this sense of relief was also fear and concern over re-injury. Laura indicated that once she considered her knee injury healed, she still worried about injuring it again.

"Sometimes," she said, “like if I'm not really paying attention to like if there's a branch or something and I step on it or a rock and I kind of tweak my leg I just get kind of nervous that it's going to set it off again.”

Laura also noted that, despite that her healthcare professional told her it was unnecessary, she continued to wear a brace on her knee out of fear that she would reinjure the knee if she was not wearing the brace. Maryn indicated that the fear of having another injury continued to impact her ability to train even months after the onset of her hip injury

“When I do my sprint workouts... Um, I don't sprint as hard as I normally would. So, I’ve learned that I have to take it down and then the next sprint workout I can, I can go more at $100 \%$. So, that first sprint workout is only about $85 \%$ because otherwise I'll be right back to where I was again.”

Fear of appearing weak. Finally, there was also evidence of fear of appearing weak to others. Participants in this study had many social connections related to running, such as teammates, training partners, coaches, and instructors, and exhibited fears of appearing weak to these important others in their lives. For example, Brad noted fear of being viewed as weak because of his stress fracture. He said, 
“Ah, I just thought that it would be viewed as weakness and that's not the way I wanted people to view/see me as.”

Maryn also indicated an awareness of trying not to appear weak to those around her

“Iinitially it's stressful and the fact that I can't do that, and when you're around a ton of people who are running that day and you can't do what you are supposed to do, it's kind of that you feel as though you're being perceived as being weak.”

General psychosocial distress. In addition to frustration and fear, many of the participants in the study indicated that they experienced general emotional distress during the injury phase of their stories. Even though participants had been dealing with their injuries for months and often perceived themselves to be recovered at the time of interviews, in telling their injury stories participants were often visibly emotional-crying, shaking, or becoming flushed. Verbally, participants reported feelings of sadness, devastation, and anger while they were injured. Jordan reported emotional distress over her injury saying,

“I was like really upset about like almost crying about it, 'cause like this sucks.”

While Laura indicated she was

"like super bummed out"

in response to her injury. For Jane the idea of not being able to complete her upcoming marathon was very upsetting for her:

“That's just absolutely devastating to me right now."

Moreover, Logan simply said,

“It sucked because I couldn’t run.”

Both the body language and the verbal communication by participants indicated a strong degree of psychosocial distress associated with their injury.

Social influence. Participants’ psychosocial responses were also influenced by important others in their lives. Participants noted that they elicited or received social support from a number of different sources throughout their injury, including running friends, family, and coaches/instructors. For example, Grace reported receiving social support from her family, indicating:

"I have a family that is really supportive and so they're praying for me and then they'll just listen”,

as well as receiving support from her coaches and teammates. Moreover, Jordan indicated that she received support from her running group, indicating that many of these people had also suffered injuries and thus could relate to what she was experiencing:

"Like we all support each other," she said, "like we'll text each other like later in the day like, hey, how's your knee feeling now?”

Notably, participants did not necessarily seek out support from healthcare professionals as we might expect considering the amount of distress — both physical and emotional—-they were experiencing. This point will be discussed further in the results related to research question 2 . 


\subsection{Research Question 2: Chronology of Experiences}

Research question 1 addressed the psychosocial responses to running injuries that persisted throughout the injury experience. In research question 2, we focused more specifically on the chronology of thoughts, feelings, and behaviors that changed systematically as the participants went through the injury process. Thematic analysis of participants' stories revealed consistent patterns of experiences prior to injury, throughout the injury, and at the outcome of the injury. A summary of themes related to research question 2 is available in Figure 1. As per the interview question, participants' experiences with injury fell into three chronological categories - pre-injury, injury onset, and outcome. Within each injury phase participants noted some unique thoughts, feelings, and behaviors associated with their injury.

Pre-injury factors. Pre-injury was defined as the period of time immediately prior to the injury onset when the participant was training for a running event but did not experience symptoms of the injury. Pre-injury factors were characterized by factors prior to injury that influenced the athletes' perceived risk of injury and response to injury.

Injury attributions. It was evident throughout the interviews that participants had carefully considered the question of why they had become injured. Overwhelmingly, participants attributed their injury to one of two factors-overtraining and/or change in running conditions or training. Kathryn, for example, attributed her injury to both overtraining and change in running conditions. Specifically she indicated going from treadmill running to outdoor running and increasing her mileage quickly.

"It [running] was never outside, it was always on a treadmill for that and then I was way over-doing it, because I'd be like... I'd never, kinda of gradually went up, I just like 'I'm gonna do six miles in the first day'. And that was a bad idea. And I did that for the first two weeks and I was seriously hurting."

Anne also attributed her injury to overtraining, noting:

"Umm, it’s not a big story I guess I was just maybe I was running too much.”

Grace and Laura thought that a change in running conditions, specifically weather conditions, was the cause of their injuries. Participants in the study were primarily from the Midwestern United States and interviews were conducted in the spring after a particularly cold and snowy winter. Participants suggested these harsh running conditions and specifically going from fall running, where the trails and sidewalks were free of ice and snow, to slippery winter conditions contributed to their injury risk. For example, Grace indicated that

"I was just doing a run and I had just like, it was like snowy outside and, um, it was icy so I felt my Achilles.”

Laura also attributed her injury to winter running conditions, indicating that

"the only thing that was kinda like different from my normal running is that we were running in snow so it was kinda slushy so that's kinda how my injury happened.”

Goal planning. Another consistent theme in the injury narratives was that participants' experiences with injury seemed to be shaped by the fact they had a goal or deadline by which time they felt they needed their injury to have healed. Specifically, participants overwhelmingly had a race or event that 
they wanted to complete within the near future. This goal seemed to be influential in participants' emotional responses to injury. For instance, Maryn indicated that her initial response to her quad injury was:

“stress because I can’t afford to take any time off of the running.”

Others worried about having their injury get better or at least being able to control their symptoms prior to the time or a race. Specifically, many participants in this study were concerned about being able to complete their first marathon. Participants noted some degree of pressure associated with training for a specific, time-sensitive running goal. Participants indicated these running goals were inflexible and may have contributed to their overtraining or change in training. For example, Logan noted that he chose to run every day rather than taking any rest days because he was trying to complete a marathon in his goal of under three hours. He reported that he thought this contributed to his injury.

Injury onset. The onset category in the chronology of injury experience is considered the period of time during which runners perceive themselves to be injured or have pain that is affecting their running.

Diagnosis and treatment. The next phase of injury stories in this study was the injury phase. Although all injuries in this study were considered microtrauma injuries, which typically have insidious onset, participants usually identified a specific day or run during which the pain became unmanageable or started to negatively impact their running. Once injuries had progressed to this point participants noted that they almost immediately began to try to figure out how to manage the injury. There was a typical pattern of how participants went about treatment of their injuries. The majority of participants did not seek formal medical treatment for their injury. Thus, they typically engaged in self-diagnosis and self-treatment of their injuries. Participants had similar patterns of treating or minimizing physical distress of the injury while being acutely aware of a "goal” or "deadline" by which they needed to be injury-free or at least able to complete a specific running event. Only three of the 10 participants in this study formally visited a healthcare professional to receive a diagnosis or treatment for their injury. More often, participants enlisted information and advice from a number of alternative sources. These sources included healthcare professionals who were friends or family members, coaches or instructors, other runners, and the Internet.

The information participants received from these sources then informed their own treatment plan for their injury. For instance, Jordan said in regard to treating her iliotibial (IT) band injury that she enlisted information from both a running coach and the Internet. She said,

"I talked to [my coach]... I told him what was going on and, like, umm, I told him I thought it was an IT band issue and he agreed with me and he just said take it easy.”

She continued to seek out information about IT band issues, but looked online.

“I remember that Monday night,” she said, “umm, I read online about foam rollers and like how you can foam roll it [IT band] out and a lot of people like who have IT band issues they'll get a foam roller and it like works magic.”

This information she received informed her use of a foam roller in treatment of her injury. Additionally, many participants noted engaging in icing, stretching, or physical therapy-type exercises in order to treat their injury or at least minimize the discomfort they were experiencing. Specifically, Laura, who was 
one of the few participants to see a physical therapist, explained that her physical therapist instructed her on how to tape her knee as well as what exercises to do. This instruction allowed her to care for her injury independently.

Continued training. Despite participants reporting a great deal of discomfort associated with their injuries and that their injuries negatively impacted their running, only one participant out of 10 indicated they took more than a week off from running between the time of their injury and their next running competition. Maryn said that even if she was told by a healthcare professional not to run, she would not take time off from running, specifically noting,

“Like, if I’m told I can’t run, that’s just not going to work.”

Kathryn indicated that she continued to run despite her injury, saying,

"I never took more than like one or two days off from running. I tried to stick with it because I figured it would be harder if I just stopped then went back to it.”

However, they did note decreasing or changing their running in order to deal with the injury while increasing cross-training activities such as biking or using the elliptical. Logan, in his explanation of why he did not take much time off, said,

“One day I felt pain so I took a few days off and then I did it, I ran again, and I still felt pain but I could push through it.”

Jordan noted modifying her running in order to deal with her injury. Specifically, she said,

“I haven’t felt good since like the injury but like I've really learned how to deal with it and like, umm, on our runs I would just like slow down.”

Brad indicated that he was in the taper phase of his marathon training when his injury occurred and therefore he was less concerned about taking time off as compared to during other times in his training.

"I reduced my mileage," he said, "I originally planned on doing for those two weeks just because I wanted to let it rest and let it recover but I didn't want to just completely stop training so I still ran at least once a week.”

Some participants indicated that cross-training aided in minimizing the distress they felt from being injured. Anne said:

“I think that would have been a big difference would be if I couldn’t do anything active, it would have meant a lot more, but I could still do something active with both those injuries so it didn’t pull me out of physical activity all together so I think that made a big difference.”

Grace noted her coach provided her with workouts that were helpful in dealing with her injury both physically and mentally. Specifically, she said,

“It's [cross-training workouts developed by her coach] been really helpful to being feeling like I still have a little bit of control of, like maintaining fitness and stuff like. So, doing workouts, that helped me cope with it a little.”

Outcome. The outcome phase of the chronology of injury is considered the point at which the runners perceived themselves to have recovered from the injury or the point at which they were at the time of 
interview. Participants having some change in training as well as learning lessons from the injury characterized this phase.

Change in training. For many participants the outcome phase was short-lived as they often reported a second injury or recurrence of the injury once they thought it had healed. Despite an early reluctance to take time off from training, many participants had made some changes in training in order to cope with their injuries. For example, Jordan said,

“I haven’t felt good since like the injury but like I’ve really learned how to deal with it and like on our runs I would just like slow down.”

Other participants noted they took a substantial break from running after their goal or deadline. Therefore, the outcome phase, for some, was not until after they had completed their goal race and had taken a break from running.

Lessons learned. Additionally, participants reported learning things from their running injury experiences, especially about perseverance as well as preventing injuries in the future. For example, Logan noted that he has developed strategies in his training in order to prevent injuries, specifically he said,

“I’m more cautious, I would say. I don’t go out and do seven miles after seven miles after seven miles anymore.”

Brad also learned a lesson about overtraining, indicating the injury

"kind of taught me that I was maybe overtraining a little bit. I was running prior to that [the injury] and just kept building up on it and at some point I figured something would happen, but I kind of hoped it would be later.”

Jane also indicated how important it was to take care of your body. Specifically she said, "Like, I want to be healthy so bad. Um, I definitely respect it [her body] more. Like you have to take care of your body”.

In addition to learning how to prevent injuries in the future, participants also shared that they felt they had learned to persevere through difficult times during their injury. Jordan indicated that she learned not to

"freak out right off the bat, like umm I guess I was really concerned with the outcome, like am I going to be able to reach my goal, like is this going to prevent me from actually doing the marathon, so I was quick to jump to the worst case scenario.”

She added that,

“I don’t know, I jumped to conclusions then I realized I need to relax about this so I guess just not panicking and not assuming the worst.”

Laura noted that she learned that she could deal with more than she thought, specifically she said, "One thing that it [the injury] taught me is like I can push through pain a lot better than I thought I could.” 
She also said,

“It also helped me to just work through an obstacle because, I don't know, I hadn’t really

had a lot of obstacles I’ve had to work around, especially in exercise.”

Jane indicated that

"I definitely think that I’ve become tougher."

Upon reflecting on their injury experience when telling their injuries stories, participants generally indicated continued positive feelings about running despite their injuries. Jane, who was training to complete a marathon, reflected that completing the marathon would mean more to her now because of her injury.

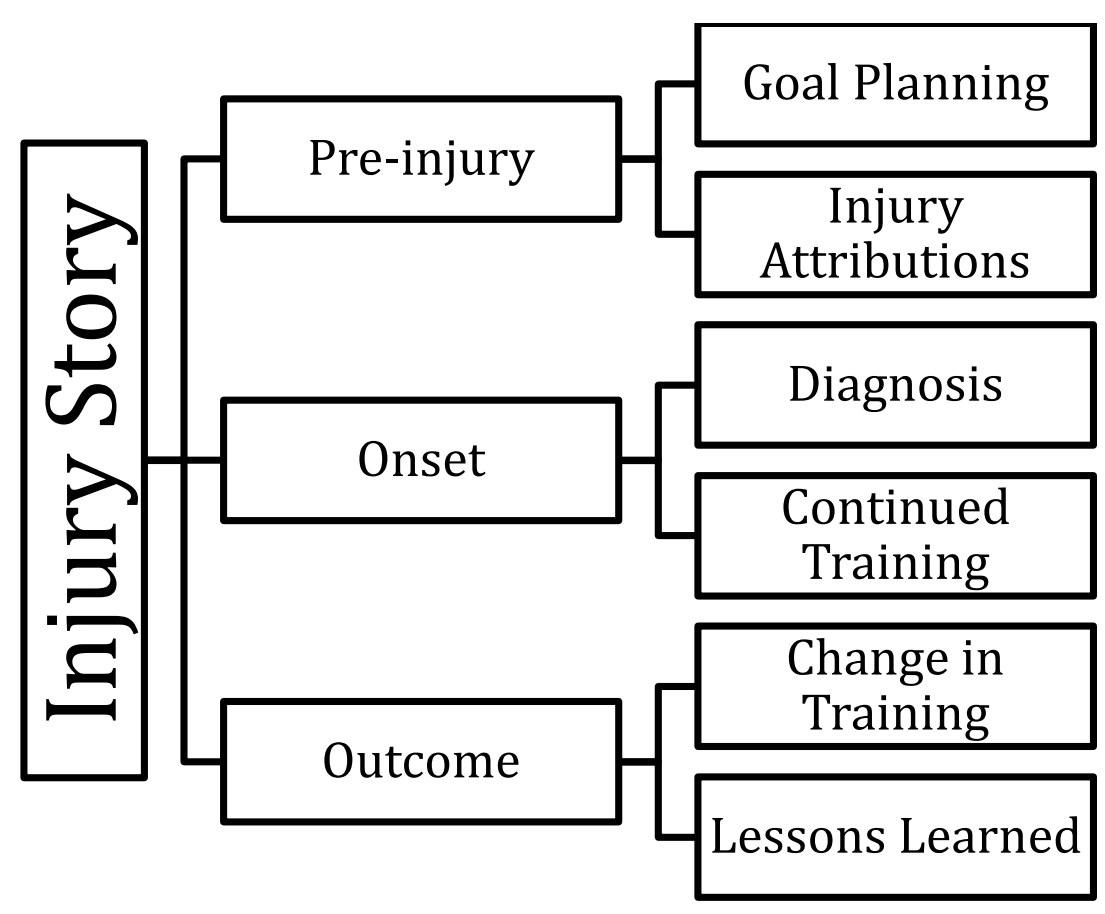

Figure 1. Summary of results related to research question 2, the chronology of responses to microtrauma running injury. The figure denotes that there were three injury phases: pre-injury, onset, and outcome, and each phase had distinct psychosocial responses.

\section{Discussion}

The purpose of the current study was to use a variation of Mishler's [20] core-narrative approach, as adapted by Brock and Kleiber [10], to examine and better understand the unique experiences and psychosocial responses of adult long-distance runners who had incurred a microtrauma injury. The use of qualitative data analysis of the 10 different interviews led to a consistent chronology of consistent cognitive, affective, and behavioral responses throughout the course of injury. In regard to the chronology of events, participants consistently responded within three distinct phases-pre-injury, injury onset, and outcome-and within each of these time points, certain themes emerged related to psychosocial responses to injury. 
Four consistent psychosocial responses were identified throughout the injury process. They included frustration due to uncertainty of the injury and recovery process, fear related to the injury failing to heal, an inability to meet a specific goal, becoming re-injured, and appearing weak, general emotional distress that included descriptors of feeling sad, devastated, and angry, and, finally, social support/social pressure as instigating factors for the onset of the negative psychosocial responses. Unlike the themes related to the chronology of experiences and responses to injury, these psychosocial responses were evident from the onset of injury throughout the recovery and overcoming of the injury.

During the pre-injury period, participants noted the importance of their running goals and discussed how those goals affected their risk of injury and response to injury. They made consistent attributions to overtraining or a change in running conditions as instigating factors for a microtrauma injury. Psychosocial factors during the onset phase included self-diagnosis and treatment for the injury as well as reports of continuing to train through the injury. During the outcome phase, participants reported changing their training as well as learning lessons as a result of their injuries. Specifically, they described they could use the lessons they had learned to get through potential future injuries or other challenges that may occur in life.

In general, findings from the current study add support to the integrated model of psychological response to sport injury [3] and to other previous research findings that athletes incurring sport injuries experience not only the physical consequences of an injury, but also the psychosocial responses surrounding the sport injury [12,21-23]. Findings from the current study provide evidence for a need to study the role of injury type to better understand the psychosocial responses across the time course of the injury process [3]. Furthermore, they confirm the initial hypothesis proposed by Flint [5]: that the progression and longevity of microtrauma injuries contribute to the psychosocial distress experienced by athletes incurring these types of injuries.

The results of this study support some of the findings from the limited research on psychological responses to microtrauma injuries, specifically with respect to social support. Participants in this study reported seeking little social support, especially from healthcare professionals. This is consistent with work by Wasley and Lox [7], who noted that athletes with microtrauma injuries are less likely to seek out social support as compared to athletes with macrotrauma injuries. Injured athletes not seeking medical attention is potentially problematic for both physical and psychosocial well-being. Not seeking formal medical care may lead athletes to make uninformed decisions, thus putting them at risk for sustained injury or additional physical harm. Further, as a number of researchers have identified healthcare professionals as a key source of social support for injured athletes [10,24-26], these athletes who isolate rather than seek assistance are missing out on other opportunities for educational support, such as strategies for the prevention of future injuries. Despite not seeking support from heath care professionals, the results suggest that long-distance runners with microtrauma injuries do rely on social support from other social domains such as friends and family.

The current findings support the notion that long-distance runners who experience a disruption in training due to an injury experience significant psychosocial distress [2] but also provide evidence of variations in psychosocial distress throughout the injury and rehabilitation process. The results of the current study add to the existing literature by indicating that a specific chronology of phases occurs during a microtrauma running injury, each with unique cognitive, affective, and behavioral responses. During each of these phases, athletes incurring a microtrauma injury experienced distinct yet consistent 
psychosocial responses. This finding is crucial for helping coaches and healthcare professionals become aware of expected responses to a microtrauma injury and to become more aware of when these responses will occur depending on the phase of the injury process. By utilizing a narrative research methodology, we were able to provide a clearer picture of the complexity of psychosocial responses to sport injuries.

The findings from this exploratory study provide important information for athletes, coaches, and practitioners. For example, the majority of participants reported that they engaged in some form of self-diagnosis rather than seeing a healthcare professional. Therefore, coaches need to be aware of this proclivity of athletes and, therefore, encourage a formal diagnosis and provide athletes experiencing microtrauma injuries with the available resources to attain professional care. Likewise, healthcare professionals aware of this tendency are encouraged to provide information through various sources regarding symptoms, treatment, and alternative activities to help these individuals deal with injuries on their own. Additionally, both coaches and healthcare professionals working with athletes who have experienced a microtrauma injury must be aware that either the nature of the injury or characteristics of the individual athlete may decrease the likelihood that s/he will take time off when injured. Compared to macrotrauma injuries that are usually visible (e.g., broken foot) and require individuals to take time off from training, microtrauma injuries are often invisible (e.g., Achilles tendonitis) and, therefore, may lead an athlete to conclude that nothing is wrong if the injury cannot be seen. Given this penchant for continued exercise and training of individuals with microtrauma injuries, both coaches and healthcare professionals need to provide athletes with alternative activities that will help them continue to meet their training and competition goals.

\section{Limitations}

Despite the additions to the literature of this study, a number of limitations exist. First, several of the participants were novices training over several months for their first competitive long-distance run as part of a coached group. Many runners felt significant internal and external pressures to achieve the final goal of competing in the targeted run, and this may have cause them to think, feel, or behave differently than if they were training on their own. Nonetheless, the use of participants from a long-distance running group helped to identify the consistent theme that individuals often feel pressure to recover from an injury or to compete while injured in order to meet a specific goal. Additionally, this sample may be different from a more general running population because of their willingness to participate in interviews about their injury. Other injured runners who did not volunteer for our study may have had different psychosocial responses to injuries, therefore the results cannot be generalized to all runners.

Second, the study sample was limited to long-distance runners with microtrauma injuries. Although the findings from the current study provide an understanding of the injury and recovery process associated with microtrauma injuries, the patterns that emerged cannot be generalized to all athletes who experience microtrauma injuries. Moreover, the participants' injuries were self-reported and self-diagnosed, so we cannot definitively determine the diagnoses or severity of each injury. However, the findings from the current study begin to provide a better understanding of the injury and recovery process and will be important to compare with future studies that may look at the injury and recovery process of individuals sustaining microtrauma injuries from training and competing in different types of sports. 
Lastly, due to the nature of qualitative studies and the use of narrative research methodology in the current study, specific causal inferences cannot be gleaned from the study's findings. Although we have made inferences throughout the paper that the participants' responses and experiences were related to microtrauma injuries, since we did not interview participants with macrotrauma injuries, we cannot definitively assert that this is the case. Regardless, the findings from the study do provide an important platform of the structure of physical and psychosocial responses to microtrauma sport injuries.

\section{Future Directions}

Findings from the current study provide an important starting point for researchers and practitioners to better understand the sport injury process for individuals who experience a microtrauma injury. Future research could strengthen the findings from this study by conducting qualitative research studies using narrative research methodology with individuals incurring microtrauma injuries from a variety of sports. This would allow for researchers to better understand the pattern of responses to microtrauma injuries and determine whether injury type does differentiate athletes' responses to injury or if response to injury has more to do with the type of sport an individual engages in. Additionally, future research using qualitative methods could compare the responses to injuries for athletes from the same sport but who differ in injury type to help better determine unique or similar responses. Lastly, once qualitative research has further confirmed that differences in response style exist depending on the type of injury an individual incurs, quantitative research could be used to solidify that athletes with microtrauma injuries score differently on measures of various psychosocial responses than athletes experiencing macrotrauma injuries.

\section{Conclusions}

The use of a narrative research methodology to study the physical and psychosocial responses of long-distance runners incurring microtrauma injuries provided a clearer picture of the complete injury process and the changes in both physical and psychosocial responses that occur at the different phases of the injury process. The findings provide further support for the integrated model of psychological response to sport injury [3] and, specifically, the importance of better understanding the role of injury type as a factor moderating the psychosocial responses of injured athletes. Rather than limiting participants to predetermined responses, the use of narrative research methodology provided the opportunity to identify unique responses to the injury process. For example, this study highlighted unique cognitive responses to injury, such as fear of taking time off from training, affective responses like frustration about the injury type, and behavioral responses like avoidance of formal medical care, that may have been missed using more structured or constrained methods of inquiry. To understand the dynamic complexities of the physical and psychosocial responses to injury, it is crucial that future research employ a variety of research techniques to test various aspects of the integrated model of psychological response to sport injury [3], as well as other models of responses to sport injuries. Additionally, it is crucial for future research to address the potentially unique psychosocial responses and needs of athletes with differing injury characteristics in order to provide the best care and support for all injured athletes. 


\section{Acknowledgments}

We thank Reed H. Steele and Ayanna N. Franklin for their important and significant contributions to this project.

\section{Author Contributions}

Hayley Russell and Diane Wiese-Bjornstal designed this study. HR collected and analyzed the data. HR and DWB wrote manuscript.

\section{Conflicts of Interest}

The authors declare no conflict of interest.

\section{References}

1. Lun, V.; Meeuwisse, W.H.; Stergiou, P.; Stefanyshyn, D. Relation between running injury and static lower limb alignment in recreational runners. Brit. J. Sport Med. 2004, 38, 576-580. doi:10.1136/bjsm.2003.005488.

2. Chan, C.S.; Grossman, H.Y. Psychological effects of running loss on consistent runners. Percept. Motor Skill. 1988, 66, 875-883. doi:10.2466/pms.1988.66.3.875.

3. Wiese-Bjornstal, D.M.; Smith, A.M.; Shaffer S.M.; Morrey, M.A. An integrated model of response to sport injury: Psychological and sociological dynamics. J. Appl. Sport Psychol. 1998, 10, 46-69. doi:10.1080/10413209808406377.

4. Wiese-Bjornstal, D.M. Psychology and socioculture affect injury risk, response, and recovery in high-intensity athletes: A consensus statement. Scand. J. Med. Sci. Sports 2010, 20, 103-111. doi:10.1111/j.1600-0838.2010.01195.x.

5. Flint, F.A. Integrating sport psychology and sports medicine in research: The dilemmas. J. Appl. Sport Psychol. 1998, 10, 83-102. doi:10.1080/10413209808406379.

6. Van Gent, R.N.; Siem, D.; van Middelkoop, M.; van Os, A.G.; Bierma-Zeinstra, S.M.A.; Koes, B.W. Incidence and determinants of lower extremity running injuries in long distance runners: A systematic review. Brit. J. Sport Med. 2007, 41, 469-480. doi:10.1136/bjsm.2006.033548.

7. Wasley, D.; Lox, C.L. Self-esteem and coping responses of athletes with acute versus chronic injuries. Percept. Motor Skill 1998, 86, 1402. doi:10.2466/pms.1998.86.3c.1402.

8. Henert, S.E. Exploring Injured Athletes' Ratings of Social Support and Use of Coping Strategies as a Function of Injury Type and Gender over the Course of Rehabilitation, Ph.D. dissertation. University of Minnesota, Minneapolis, MN, USA, 2000. ProQuest (AAT 9966232).

9. Bianco, T. Social support and recovery from sport injury: Elite skiers share their experiences. Res. Q. Exercise Sport 2001, 72, 376-388. doi:10.1080/02701367.2001.10608974.

10. Brock, S.C.; Kleiber, D.A. Narrative in medicine: The stories of elite college athletes' career-ending injuries. Qual. Health Res. 1994, 4, 411-430.

11. Podlog, L.; Eklund, R.C. High level athletes' perceptions of success in returning to sport following injury. Psychol. Sport Exercise 2009, 10, 535-544. doi:10.1016/j.psychsport.2009.02.003. 
12. Tracey, J. The emotional response to the injury and rehabilitation process. J. Appl. Sport Psychol. 2003, 15, 279-293. doi:10.1080/104132003902137924.

13. Bleakley, A. Stories as data, data as stories: Making sense of narrative inquiry in clinical education. Med. Educ. 2005, 39, 534-540. doi:10.1111/j.1365-2929.2005.02126.x.

14. Lieblich, A.; Tuval-Mashiach, R.; Zilber, T. Narrative Research: Reading, Analysis, and Interpretation; Sage Publications, Inc.: Thousand Oaks, CA, USA, 1998.

15. Bailey, P. The dyspnea-anxiety-dyspnea cycle_COPD patients' stories of breathlessness: "It's scary/When you can't breathe.” Qual. Health Res. 2004, 14, 760-778. doi:10.1177/1049732304265973.

16. Borkan, J.M.; Quirk, M.; Sullivan, M. Finding meaning after the fall: Injury narratives from elderly hip fracture patients. Soc. Sci. Med. 1991, 33, 947-957. doi:10.1016/0277-9536(91)90265-E.

17. Patton, M.Q. Qualitative Research and Evaluation Methods; Sage Publications, Inc.: Thousand Oaks, CA, USA, 2002.

18. Eatough, V.; Smith, J. Interpretative phenomenological anaylsis. In The Sage Handbook for Qualitative Research in Psychology; Willig, C., Stainton, W., Eds.; Sage Publications, Inc.: Throusand Oaks, CA, USA, 2008.

19. Tesch, R. Qualitative Research Analysis Types and Software Tools; Falmer Press: New York, NY, USA, 1990.

20. Mishler, E.G. The analysis of interview-narratives. In Narrative Psychology: The Storied Nature of Human Conduct; Sarbin, T.R., Ed.; Praeger: New York, NY, USA, 1986; pp. 233-255.

21. Jevon, S.M.; Johnston, L.H. The perceived knowledge and attitudes of governing body chartered physiotherapists towards the psychological aspects of rehabilitation. Phys. Ther. Sport 2003, 4, 74-81.

22. McDonald, S.A.; Hardy, C.J. Affective response patterns of the injured athlete: An exploratory analysis. Sport Psychol. 1990, 4, 261-274.

23. Walker, N.; Thatcher, J.; Lavallee, D. Psychological responses to injury in competitive sport: A critical review. J. R. Soc. Promo. Health 2007, 127, 174-180. doi:10.1177/1466424007079494.

24. Mann, B.J.; William, G.A.; Indelicato, P.A.; O’Neill, D.F.; George, S.Z. A survey of sports medicine physicians regarding psychological issues in patient-athletes. Am. J. Sport Med. 2007, 35, 2140-2147. doi:10.1177/0363546507304140.

25. Russell, H.; Tracey, J. What do injured athletes want from their health care professionals? Int. J. Athl. Ther. Train. 2011, 16, 18-21.

26. Tracey, J. Inside the clinic: Health professionals’ role in their clients’ psychological rehabilitation. J. Sport Rehabil. 2008, 17, 413-431.

(C) 2015 by the authors; licensee MDPI, Basel, Switzerland. This article is an open access article distributed under the terms and conditions of the Creative Commons Attribution license (http://creativecommons.org/licenses/by/4.0/). 